\title{
A Review on Post and Core
}

Dr. Himanshi Kalra MDS ${ }^{1^{*}}$, Dr. Urvashi Sukhija ${ }^{2}$, Dr. Reena roy Rassawet ${ }^{3}$, Dr. Varsha Rani MDS ${ }^{4}$

${ }^{1}$ Post Graduate Student, Deptt. of Prosthodontics, MMCDSR, Mullana, Ambala Haryana, India

${ }^{2}$ Professor, Deptt. of Prosthodontics, MMCDSR, Mullana, Ambala Haryana, India

${ }^{3}$ Deptt. of Prosthodontics, MMCDSR, Mullana, Ambala Haryana, India

${ }^{4}$ Post Graduate Student, Deptt. of Prosthodontics, MMCDSR, Mullana, Ambala Haryana, India

DOI: $\underline{10.36347 / \text { sjds.2020.v07i03.002 }}$

| Received: 27.02.2020 | Accepted: 05.03.2020 | Published: 10.03.2020

*Corresponding author: Dr. Himanshi Kalra

Abstract

The number of endodontic procedures has increased steadily in the past decade with highly predictable results. Therefore, restoration of teeth after endodontic treatment is becoming an integral part of the restorative practice in dentistry. When restoring an endodontically treated tooth, the first step is to assess the level of predictability involved in the restoration. Proper restoration of ET teeth begins with a good understanding of their physical and biomechanical properties, anatomy, and a sound knowledge of the endodontic, periodontal, restorative and occlusal principles. A thorough understanding of posts is necessary to make the right selection, as there are so many choices available. Finally, the choice of core material and the final restoration are important in achieving long-term clinical success. This review summarises the indications, contraindications for different post and core materials, principles of canal preparation as well as advantages and disadvantages of custom and pre- fabricated post.

Keywords: Endodontically treated tooth, custom post, pre- fabricated post, biomechanical considerations, canal preparation.

Copyright @ 2020: This is an open-access article distributed under the terms of the Creative Commons Attribution license which permits unrestricted use, distribution, and reproduction in any medium for non-commercial use (NonCommercial, or CC-BY-NC) provided the original author and source are credited.

\section{INTRODUCTION}

In earlier days, extraction was the suggested treatment of choice for most teeth that were grossly carious, but today, the focus of dental therapy has shifted to a more conservative approach. The overwhelming success of endodontic therapy has allowed for not only the restoration of such teeth, but such teeth has also reinstated it as a long-term functional unit inside the oral cavity. The techniques and guidelines of how and when to restore endodontically treated teeth has evolved from clinical tradition and anecdotal descriptions [1].

The goal of prosthodontic and restorative dentistry is to replace missing tooth structure, maintain function and aesthetics and protect against fracture or infections [2]. Endodontic treatment removes the vital contents of the canal, leaving the tooth pulpless and resulting in teeth with calcified tissues and significantly less moisture than that of vital teeth[1]. This was thought to considerably weaken the tooth structure making it significantly more susceptible to fracture under masticatory forces. The treatment concept is based upon various factors so that the strategic architectural aspects produce the greatest positive impact on the strength of a pulpless tooth which can then, be restored and reinforced to sustain fracture when subjected to vertical and lateral forces. Currently, the treatment decision is based on the vastly expanded knowledge about newer materials and techniques in the field of endodontics and prosthodontics. Whatever might be the choice of restorative procedure selected for an endodontically treated tooth, success can only be achieved when the choice of technique best meets the needs of the individual clinical diagnosis - specifically, the needs of the individual diseased tooth and the clinical use for which it is intended[3].

\section{CRITERIA FOR SELECTION}

Formulation of a precise treatment plan is based on the consideration of the differences in endodontically treated teeth from intact vital teeth. Special techniques are usually necessary to restore endodontically treated teeth as their tooth structure is considerably lost. The selection of specific materials and technique for the restoration of endodontically treated teeth is influenced by the changes that accompany root canal therapy [2]:

- The amount of remaining tooth structure

- Physical changes in tooth structure 
- The anatomic position of the tooth

- The occlusal forces on the tooth

- The restorative requirements of the tooth

- The esthetic requirements of the tooth

\section{Amount of remaining tooth structure}

The amount of remaining tooth structure forms one of the most important factors to be considered during the restoration of endodontically treated teeth. Loss of tooth structure for an endodontically treated tooth may vary from a minimal endodontic access preparation to very extensive damage that endangers the longevity of the tooth. The amount of remaining sound coronal tooth structure is far more significant for the long term prognosis of the restored tooth than any other factor because no restorative material can act as a replacement for intact dentin [2]. Access opening procedure destroys the roof of the pulp chamber which provided structural integrity to the coronal dentin and greater flexion under function. However, it has been shown that endodontic procedures reduce tooth stiffness by only $5 \%$, attributed primarily to access opening, whereas tooth structure removal in a mesio-occlusodistal (MOD) preparation reduces tooth stiffness by $60 \%[2,4]$.

\section{Physical changes in tooth structure}

Properties of dentin get irreversibly altered due to endodontic procedures. Changes in collagen crosslinking and dehydration of dentin result in a $14 \%$ reduction in strength and toughness in endodontically treated molars [1]

\section{Nature of dentin toughness}

Dentine is a natural, hydrated, mineralized hard tissue that forms the major bulk of a tooth. Mature dentine is a composite material made up of an organic fraction (30 wt\%), which is mainly collagen and an inter-penetrant inorganic fraction $(60 \mathrm{wt} \%)$ and water (10 wt $\%)$. An optimum balance between stiffness and dynamic toughness is crucial for the mechanical stability of a biological structure such as dentine [5].

\section{Role of moisture}

The interaction of water and dentin matrix occurs in a well-defined manner [5]. In hydrated dentin, water is said to act as a plasticizer, keeping the matrix soft and pliable. The collagen fibrils of dentin collagen are made up of smaller microfibrils separated by spaces filled with water [5]. Dehydration leads to the loss of these interfibrillar spaces and shrinkage of the overall diameter of the fibrils.

\section{Visco-elastic characteristics in dentin include}

- An increase in strain with time when stress is held constant (creep)

- A decrease in stress with time when strain is held constant (stress relaxation)

- The stiffness depends on the rate at which the load is applied
- During rolling, frictional resistance occurs

- Loss of free water compromises all characteristics of visco-elastic behaviour[5]

\section{TOOTH POSITION AND OCCLUSAL FORCES Considerations for Anterior teeth}

Anterior teeth are subjected to flexural forces and tensile forces more than they are subjected vertical compressive forces. These forces are delivered primarily to the facial and lingual surfaces of the teeth. Therefore, the incorporation of an internal post usually does not help prevent fracture [6]. Anterior teeth are placed away from the line of fulcrum line and therefore have a lesser amount of force acting over them.

According to a study by Sorensen and Martinoff [7], there was no significant difference between the success achieved with anterior pulpless teeth that had received crowns and those that did not. Another study by Lovdahl and Nicholls [8] found that intact endodontically treated central incisors were three times as resistant to fracture as teeth that had been restored with dowel cores.

In discoloured teeth with endodontic treatment, bleaching is the preferred option over a full crown. Reduction of the tooth structure for placement of the crown would lead to further loss of tooth structure in addition to the loss of tooth structure accompanying root canal procedure and is not recommended.

\section{Considerations for Posterior teeth}

The anatomy of posterior teeth is such that the grooves naturally divide the occlusal table of the tooth. Plus, compressive forces are directed over these teeth which try to wedge the cusps apart.

Hence, the minimum required restoration for an endodontically treated posterior tooth is an MOD onlay. The presence of a crown encircles the tooth and helps sustain the wedging masticatory forces. This is especially true for maxillary premolar teeth whose occlusal anatomy favours wedging forces and cuspal deflection.

A full coverage restoration is usually indicated in posterior teeth unless it has a very conservative occlusal access opening and the teeth is not subjected to heavy occlusal stresses, like in a mandibular premolar where the occlusal table is not wide and is not subjected to heavy masticatory forces.

\section{RESTORATIVE REQUIREMENTS}

The design for the restoration is based on the amount and direction of forces that the tooth (and restoration) will sustain. There will be minimal masticatory forces acting on the tooth and restoration if the tooth is an anterior tooth. Heavy compressive forces will be subjected to a posterior tooth while additional horizontal or torquing forces will be applied to the tooth 
if it is going to act as an abutment for fixed or removable partial denture prosthesis. The selection of restorative components is aimed at providing the best protection against leakage from caries and fracture [8].

\section{ESTHETIC REQUIREMENTS}

The incisors, canines, premolars and often the maxillary first molars along with the surrounding gingiva constitute the aesthetic zone of the mouth. The selection of the restorative components for these teeth should therefore also consider the aesthetic demand in each position. Aesthetic restorative components include tooth-coloured posts, composite resins or ceramic cores, cements and ceramic crown materials [8].

\section{Biomechanical considerations}

The intact natural tooth is found to experience flexing or bending stress when biting forces act on it. In a tooth under eccentric load, the compressive stresses along one side are substantially higher in comparison with the tensile stresses along the other side in the facio-lingual direction of the root. This increased propensity to compressive stresses in comparison with tensile is due to the shape and angulation of the tooth and supporting bone reactions rather than the eccentricity of loading (Fig. 4.3.1)[9].

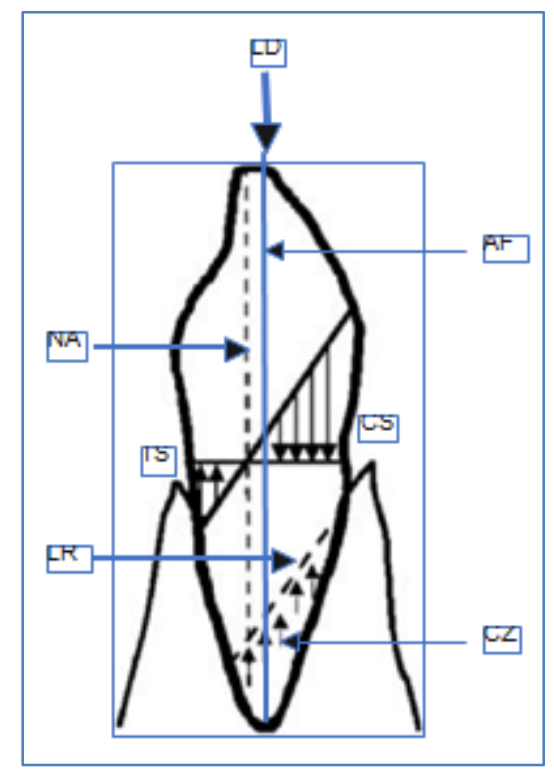

LD-Load CS-Compressive stress

TS-Tensile stress NA-Neutral axis along which stress is zero

CZ-Compressive zone AF-Axis of force

LR- Line resulting from the reactant stresses produced by the initial contact of tooth with supporting bone

The stress distribution pattern in a tooth restored using post, core, and crown is distinctly dissimilar to that of an intact tooth. In a post-core restored tooth, the 'post-core-crown-tooth system' bends or flexes as a single unit during mastication. This difference in the 'flexing pattern' of a post-core restored tooth in comparison with a normal intact tooth may be a suggested cause for periodontal bone loss in teeth with metal post [10]. The key differences between intact tooth and tooth restored using post-core are (1) occurrence of regions of stress concentration and (2) increase in the tensile stresses produced within the remaining tooth structure of a post-core restored tooth. The stress concentration intensity and tensile stresses have been noted to increase significantly when biting loads are angled away from the long axis of the tooth [11]. The factors responsible for this dissimilar stress distribution pattern in a post-core- restored teeth are (1) the greater stiffness of the endodontic post and core restoration, (2) the angulation of the post with respect to the line of action of occlusal load, and (3) increased flexure of the remaining reduced tooth structure. These factors would result in regions of stress concentration and high tensile stresses in the remaining tooth structure.

\section{Custom-cast post $\&$ core}

Cast metal posts were considered a standard due to their long successful clinical history. They have reported a high success rate. Custom cast posts eliminate the problem of bonding of post with core. They can also be easily removed in cases of retreatment. However, they require more chair time than other posts and involve laboratory procedures. They are therefore, expensive. They do not perform as well as other post systems and therefore are no longer widely used. Another disadvantage is that they require temporization. This also increases the possibility of the contamination of the root canal system.

\section{PREFABRICATED POST SYSTEMS}

Prefabricated metal posts have been widely used for the past 20 years. They can be placed easily and quickly, and a core can be added and prepared at the same appointment. They are quite strong which allows for the placement of thin posts. They can usually be removed if retreatment is necessary. Prefabricated posts are available in active or passive forms. Passive forms are recommended in most cases. A few cases require the use of active posts, primarily in short teeth where retention is minimal. However, due to their greater potential to cause root fractures and difficulty in removal, passive posts are usually preferred for most clinical situations [12].

Among rigid posts, zirconia is the stiffest, and stainless steel is stiffer than titanium alloy. Pure titanium is the least stiff of all rigid posts [2]. Zirconia posts are highly aesthetic posts and very rigid. However, they are also very brittle. Attachment of the core to the post can be a problem with zirconia posts. Being extremely hard, they need to be ground out of the canal with a bur which is a tedious and dangerous procedure. This is a significant problem if retreatment is needed [12]. 
Rigid posts and Resistance to Root Fracture

Root fracture is a catastrophic untreatable problem that results in loss of the tooth and any associated restorations.

Rigid post systems were traditionally designed to protect tooth structure from fracture by dissipating functional force along the length of the root and the periodontal structures. This is especially so in teeth with minimal remaining tooth structure [2].

Classic in vitro studies of metal posts tested in teeth without crowns found increased retention and decreased root fracture in posts that were longer and had parallel sides and serrated surface. However, this difference disappeared when the teeth were crowned with an adequate ferrule [2].

\section{Rigid posts and Structurally Compromised Teeth:}

Another role of post and core is to protect the crown margins from deformation under force and thereby protect the apical seal from coronal leakage. Coronal leakage originating in areas of margin deformation can reinfect the root canal system and lead to failure of endodontic treatment [2]. The seal provided by the post/core/crown complex is especially important for teeth with minimal remaining tooth structure. Flexion of the post, distortion of the margins, and micro-movement of the core are all detrimental to a sealed endodontically treated tooth. The restored tooth, consisting of tooth structure, post, core, cement and crown, should not flex or bend under functional loads, and the restorative components must preserve a sealed restoration. This is especially critical in damaged teeth with little remaining tooth structure at the margin [2].

Nonaxial shear, tensile, and compressive forces from biting, clenching, bruxism can be transmitted to a post in the root. Force concentration in the root predisposes the root to fracture, especially in structurally weak teeth. Rigid posts do not bend or fracture easily. In such unfavourable too structure-force combinations, a stiff post may allow restoration of a very badly damaged tooth for a time. Otherwise, the prognosis is poor and extraction should be considered [2].

\section{Non-rigid Post Systems}

Non-rigid posts are designed to have physical properties more similar to those of dentin than rigid posts. They are composed of glass, quartz or carbon fibers embedded in a resin matrix. In structurally sound teeth, non-rigid posts flex with the tooth under functional forces, thus reducing the transfer of force to the root and reducing the risk of root fracture. In structurally compromised teeth, which lack cervical stiffness from dentin, excessive post flexion can be detrimental to the marginal seal [2].
Carbon fiber posts are black, and can reflect through the gingiva, tooth structure or ceramic restorations. These posts are appropriate for teeth to be restored with gold or porcelain fused to metal crowns.

Glass and quartz fiber posts are translucent or white; these aesthetic options enhance ceramic restorations. Some glass fiber posts transmit curing light to the internal area of the root, which allows the use of dual-cure adhesive cements. The amount of light transmitted varies significantly among fiber posts, from less than $0.1 \mathrm{~mW}$ to more than $2 \mathrm{~m} \mathrm{~W}$ [2]. Light transmitting posts and bonded composite have been shown to reinforce weak roots with flared canals, providing increased fracture resistance [2]. As these posts do not require post space preparation, they preserve the integrity and the strength of shape of the canal but do not increase the risk of root fracture. Fiber posts have little to moderate radio-opacity and may be difficult to visualize in radiographs when cemented within dentin [2].

Fiber posts are adhesively bonded to the root. Carbon fiber and fiber-glass reinforced composite posts with bonded retention therefore, may not need to be as long as traditional posts. Retrievability is an important feature of endodontic posts, and fiber posts are easily removed for endodontic retreatment [2].

\section{Non-rigid posts and Resistance to Root Fracture}

Occlusal forces are transferred through the core and the post and ultimately dissipated along the length of the root. Ideally, the more posts, cements and restorative materials behave like dentin, the less the force is concentrated between the components and the root during function. Fiber posts have a lower modulus of elasticity than rigid posts of metal or zirconia.

The primary benefit of resilient posts with a lower modulus of elasticity is protection of the root from fracture through reduction of the transfer of forces through the post to the root. This post flexibility is beneficial for teeth with more than 3 to $4 \mathrm{~mm}$ of remaining axial dentin, which provides cervical stiffness to the tooth/post/core complex.

A significantly higher rate of root fracture was found in teeth restored with stiffer zirconium posts than in quartz fiber or carbon fiber posts [2]. The primary mode of failure of fiber posts has been reported to be decementation.

\section{Non-rigid posts and Structurally Compromised Teeth}

Posts and their associated core/crown complex undergo repeated lateral forces in clinical function. The physical property of non-rigidity that protects against root fracture can be detrimental in structurally compromised teeth that lack cervical stiffness from dentin walls and a crown ferrule. For a damaged tooth 
that is to be restored with a non-rigid post, 3 to $4 \mathrm{~mm}$ of cervical tooth structure must be remain to allow creation of a restoration as a whole that is resistant to flexion. Teeth with $25 \%$ or more of the tooth structure present can be restored with a non-rigid post because the cervical tooth structure itself resists lateral flexion. Teeth with minimal tooth structure above the tissue for a ferrule need additional cervical stiffness from a more rigid post to resist distortion from force. In the absence of cervical tooth structure, excessive flexibility can result in micro-movement of the core and coronal leakage [2].

All fiber posts are composed of glass, carbon or quartz fibers embedded in a matrix. The type, volume and uniformity of the fibers and the matrix have a bearing on the fracture resistance of the post. As in any physical structure, the presence of cracks, voids or sharp edges causes stress concentration that can be locally much higher than the overall stress of the structure, leading to fracture at lower levels of force. Clinically, matrix disintegration leaves intact, but excessively flexible fibers and a failed, highly mobile core and crown [2].

Non-rigid posts are not recommended for compromised teeth with very little tooth structure remaining above the tissue because the crown margin should engage at least 2 to $3 \mathrm{~mm}$ of the axial wall. If a post has the same modulus of elasticity as the root but is much thinner in diameter, it will flex more under a load. This may cause leakage under the crown and build-up. Post flexure under occlusal loads can result in micromovement of the core, disruption of the cement seal, and leakage or loss of the core and the crown. Preliminary failure of posts is clinically undetectable, yet it allows leakage, leading to caries or bacterial recontamination of the canal [13].

\section{Retrieval of post \& core system}

Post and cores had an average absolute failure rate of $9 \%$ ( $7 \%$ to $14 \%)$ when a data from eight studies with an average length of study being carried out for 6 years was compared. The most common causes of postand-core failure are post loosening and tooth fracture. The next most common causes for failure were found to be due to apical abscesses and carious lesions [7-14]. Several main causes of failure of post-retained restorations have been identified, including: recurrent caries, endodontic failure, periodontal disease, post dislodgement, cement failure, post-core separation, crown-core separation, loss of post retention, core fracture, loss of crown retention, post distortion, post fracture, tooth fracture, and root fracture. Also, corrosion of metallic posts has been proposed as a cause of root fracture [15].

Many a times, these failures in post-and-core therapy might necessitate the removal of the older post; perform endodontic retreatment followed by subsequent restoration. It has been found that fiber posts can be easily retrieved from the root canal without damaging the surrounding tissues. However, other posts like the zirconium dioxide posts are virtually impossible to remove with the currently available technologies. Various post removal kits available with the respective prefabricated post systems are efficient, but diamonds and ultrasonics were found to be more effective [16]. The techniques \& instruments currently available to remove a post \& core include Masserann technique, The Little giant post puller Kanematsu dowel removing pliers, S.S White post extractor, Post puller, Gonon post removing system, Saca Pino post extractor and many other ultrasonic and drilling systems.

\section{CONCLUSION}

When restoring an endodontically treated tooth, the first step is to assess the level of predictability involved in the restoration. Proper restoration of ET teeth begins with a good understanding of their physical and biomechanical properties, anatomy, and a sound knowledge of the endodontic, periodontal, restorative and occlusal principles. A thorough understanding of posts is necessary to make the right selection, as there are so many choices available. Finally, the choice of core material and the final restoration are important in achieving long-term clinical success. Posts do not strengthen ET teeth and should not be used in them routinely. The main function of a post is for the retention of a core if there is insufficient tooth substance left to support the coronal final restoration. The reason that many different types of posts with different designs and materials are available is because they all have certain strengths and weaknesses.

\section{REFERENCES}

1. Franklin S. Weine. Endodontic Therapy. $6^{\text {th }}$ Ed. Mosby:546-584.

2. Hargreaves, Cohen. Cohen's Pathways of the Pulp. $10^{\text {th }}$ Edition. Elsevier:786-821.

3. William Cheung. A review of the management of endodontically treated teeth. J Am Dent Assoc; 136:611-619.

4. Charles J. Goodacre, Joseph Y. K. Kan. Restoration of Endodontically Treated Teeth. Ingle JI, Bakland LK, Baumgartner JC. Ingle's Endodontics. $5^{\text {th }}$ Edition. 913-947.

5. Charles J. Goodacre, Joseph Y. K. Kan. Restoration of Endodontically Treated Teeth. Ingle JI, Bakland LK, Baumgartner JC. Ingle's Endodontics. 6th Edition. 1432-1470.

6. Rosenstiel, Land, Fujimoto. Contemporary Fixed Prosthodontics. 4th edition. Elsevier:336-378.

7. Sorensen JA, Martinoff JF. Clinically significant factors in dowel design. J Prosthet Dent. 1984;52:28-35.

8. Lovdahl PE, Nicholls JI. Pin-retained amalgam cores vs. cast-gold dowel-cores. J Prosthet Dent. 1977;38:507-514. 
9. McKerracher PW. Rational restoration of endodontically treated teeth. I. Principles, techniques and materials. Aus Dent J. 1981;26:205.

10. Felton DA. Threaded endodontic dowels: effect of post design on incidence of root fracture. J Prosthet Dent. 1991;65:179.

11. Kane JJ. Fracture resistance of amalgam coronalradicular restorations. J Prosthet Dent 1990;63:607.

12. Mendoza DB, Eakle WS. Retention of posts cemented with various dentinal bonding cements. J Prosthet Dent. 1994;72:591.
13. Machtou P, Sarfati P, Cohen AG. Post removal prior to retreatment. J Endod. 1989;15(11):552-4.

14. Gesi A, Magnolfi S, Goracci C, Ferrari M. Comparison of two techniques for removing fiber posts. J Endod. 2003;29(9):580-2.

15. Gettleman BH, Spriggs KA, ElDeeb ME, Messer HH. Removal of canal obstructions with the endo extractor. J Endod. 1991;17(12):608-11.

16. Altshul JH, Marshall G, Morgan LA, Baumgartner JC. Comparison of dentinal crack incidence and of post removal time resulting from post removal by ultrasonic or mechanical force. $\mathrm{J}$ Endodon. 1997;23(11):683-6. 